\title{
Conduite avec facultés affaiblies après la légalisation du cannabis à usage récréatif
}

\author{
Sarah B. Windle MSP, Crystal Sequeira MScA, Kristian B. Filion PhD, Brett D. Thombs PhD, Pauline Reynier MSc, \\ Roland Grad MD MSc, Carolyn Ells PhD, Mark J. Eisenberg MD MSP
}

Citation : CMAJ 2021 April 6;193:E481-5. doi : 10.1503/cmaj.191032-f

Voir la version anglaise de l’article ici : www.cmaj.ca/lookup/doi/10.1503/cmaj.191032; voir l'article connexe ici : www.cmajopen.ca/ lookup/doi/10.9778/cmajo.20200155

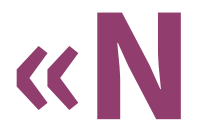

e conduis pas gelé. » C'est le message que le gouvernement canadien a envoyé à tous les conducteurs après la légalisation du cannabis à usage récréatif en octobre 2018. Toutefois, l'association entre l'utilisation légale du cannabis à cette fin et la conduite avec facultés affaiblies n'est pas claire. Même si la consommation de cannabis a le potentiel d'altérer substantiellement les habiletés psychomotrices et la fonction cognitive (réduction de l'efficacité aux tests de poursuite critique et aux tâches d'attention partagée et augmentation du temps de réaction et de la déviation de la trajectoire ${ }^{1}$ ), l'influence du cannabis sur les tâches liées à la conduite varie selon les individus, la dose et le mode de consommation, ainsi que le temps écoulé entre la consommation et la conduite ${ }^{2}$. Certaines études ont établi une association entre l'utilisation du cannabis et la conduite avec facultés affaiblies, que ce soit sur des simulateurs de conduite, sur un circuit fermé ou dans le cadre d'études épidémiologiques ${ }^{3}$, mais d'autres n'ont pas mis en évidence de tels liens ${ }^{4}$. En outre, les conclusions des études sont souvent influencées par des facteurs de confusion comme la consommation de cannabis avec d'autres substances licites (p. ex., tabac, alcool) et illicites (p. ex., opioïdes, méthamphétamine) ${ }^{3}$, ce qui rend plus difficile l'estimation de la contribution relative de la consommation de cannabis aux accidents de la route. Conséquemment, cela complique l'élaboration d'une approche optimale pour renseigner les professionnels de la santé et le public au sujet des effets du cannabis et décourager la conduite sous l'influence du cannabis.

Nous analyserons ici l'association entre la légalisation du cannabis à usage récréatif et les taux d'accidents de la route mortels et nous discuterons des implications des données probantes existantes pour les politiques canadiennes et les pratiques médicales.

\section{Que sait-on de la conduite après consommation de cannabis au Canada?}

Selon les données d'un sondage de Statistique Canada, la proportion de Canadiens ayant déclaré avoir consommé du cannabis au cours des 3 mois précédents aurait connu une hausse de $14 \%$ à $17 \%$ entre 2018 (avant la légalisation) et $2019^{5}$. Même si

\section{POINTS CLÉS}

- Selon l'analyse des données, la légalisation du cannabis à usage récréatif dans certains États américains pourrait être associée à une augmentation légère, mais significative du nombre d'accidents de la route mortels et de décès associés, ce qui, si on extrapole les conclusions au contexte canadien, pourrait signifier une hausse de 308 décès associés à un accident de la route chaque année.

- Les mesures visant à prévenir la conduite avec facultés affaiblies par le cannabis au Canada incluent l'application de limites fédérales quant à la conduite sous l'effet du tétrahydrocannabinol ( $\geq 2 \mathrm{ng} / \mathrm{mL}$ ) assorties d'amendes, l'utilisation de mesures et d'outils pour détecter l'affaiblissement des facultés par le cannabis et une campagne de publicité sur les risques de la conduite avec facultés affaiblies par le cannabis.

- Les professionnels de la santé ont la possibilité de renseigner leurs patients au sujet de l'utilisation plus sécuritaire des produits dérivés du cannabis, ce qui inclut leur conseiller de ne pas conduire après avoir consommé du cannabis (surtout en combinaison avec de l'alcool), et de leur suggérer d'attendre au moins 6 heures avant de prendre le volant.

on tient compte du fait que les répondants pourraient être plus susceptibles de déclarer leur utilisation du cannabis depuis sa légalisation ${ }^{6}$, ces données laissent entendre que l'utilisation globale aurait augmenté depuis la légalisation. Parmi les consommateurs récents de cannabis ayant un permis de conduire, $13 \%$ ont dit avoir conduit dans les 2 heures suivant leur consommation $^{5}$. Cette proportion semble être restée stable depuis la légalisation, mais le nombre absolu de personnes ayant déclaré avoir conduit peu après avoir pris du cannabis est passé de 573000 à 622000 , en raison de la hausse du nombre global d'utilisateurs 5 . De plus, près de $20 \%$ des utilisateurs ayant déclaré avoir conduit après avoir consommé du cannabis ont également déclaré avoir consommé de l'alcool en même temps ${ }^{5}$.

Une analyse basée sur les données d'un sondage de 2012 a estimé que les collisions associées à la consommation de cannabis au Canada coûtaient annuellement 1,1 milliard de dollars 
(intervalle de confiance [IC] à 95\%, 37 millions à 29 milliards de dollars) en coûts sociétaux et économiques, les conducteurs de 34 ans et moins étant responsables de la majeure partie de ces coûts ${ }^{7}$. Les auteurs ont estimé qu'annuellement, il survient 75 décès (IC à 95\% 0-213), 4407 blessures (IC à 95\% 20-11549) et 7794 collisions ayant entraîné des dommages matériels seulement (IC à 95\% 3107-13086). Cependant, ces estimations dénotent un manque de clarté en ce qui concerne les effets directement attribuables au cannabis sur les accidents de la route ${ }^{7}$.

\section{Quelle a été l'incidence de la légalisation du cannabis à usage récréatif sur les accidents de la route ailleurs dans le monde?}

Nous avons publié une analyse des données recueillies de 2007 à 2018 dans les États américains qui ont légalisé le cannabis à usage récréatif ${ }^{8}$ pour tenter d'extrapoler l'impact potentiel de la légalisation sur le bilan routier au Canada. Pour notre étude, nous avons recensé le nombre annuel de collisions fatales et de décès associés de 2007 à 2018 à partir du US Fatality Analysis Reporting System pour les 11 États où le cannabis à usage récréatif a été légalisé avant 2019 (en date de janvier 2021, l'utilisation du cannabis à des fins récréatives avait été légalisée dans 15 États et dans le District de Columbia) et nous avons tenté de déterminer s'il y avait une association entre la légalisation du cannabis à usage récréatif et les collisions mortelles. Nous avons comparé les taux pour chaque État avant et après la légalisation, puis nous avons effectué une méta-analyse des estimations pour tous les États. Nous avons conclu que la légalisation était associée à une augmentation des taux de collisions fatales (rapport des taux d'incidence [RTI] 1,15; IC à 95\% 1,06-1,26) et de décès associés (RTI 1,16; IC à $95 \% 1,06-1,27)^{8}$. Nos observations semblent indiquer que la légalisation du cannabis à l'échelle des États-Unis pourrait entraîner 4843 décès additionnels chaque année à la suite d'un accident de la route, soit une augmentation de $16 \%$. Au Canada, 1922 décès à la suite d'un accident de la route ont été signalés en $2018^{9}$; une augmentation relative de $16 \%$ correspondrait à 308 décès additionnels chaque année.

Des études antérieures ont aussi cherché à établir une possible association entre la légalisation du cannabis à des fins récréatives et les accidents de la route mortels. Une étude de 2016 a conclu que, dans l'État de Washington, la proportion de conducteurs impliqués dans une collision mortelle qui avaient obtenu des résultats positifs au dépistage du tétrahydrocannabinol (THC) avait environ doublé (passant de $8 \%$ à $17 \%$ ) dans l'année suivant la légalisation ${ }^{10}$. Même si la simple présence de THC ne signifie pas nécessairement que les facultés sont affaiblies, les résultats suggèrent à tout le moins qu'un plus grand nombre de conducteurs avaient consommé du cannabis après sa légalisation. Les auteurs d'une étude de 2019 ont comparé l'évolution du nombre d'accidents de la route mortels dans 3 États ayant légalisé le cannabis à des fins récréatives (Colorado, Washington et Oregon) par rapport aux États voisins où le cannabis n'avait pas été légalisé ${ }^{11}$; ils ont constaté une augmentation ponctuelle des accidents mortels au cours de la première année suivant la légalisation, ce que les auteurs ont attribué à une possible réaction "festive » à la légalisation ou à une augmentation du nombre d'utilisateurs de cannabis inexpérimentés, ou les deux. Ils ont aussi formulé l'hypothèse qu'une réduction subséquente du nombre d'accidents mortels pourrait résulter du fait que les conducteurs optent pour le cannabis plutôt que pour l'alcool.

Une autre étude de 2019 a aussi comparé les collisions fatales après la légalisation du cannabis à usage récréatif au Colorado et dans l'État de Washington ${ }^{12}$, montrant une tendance à la hausse, mais non significative du nombre collisions mortelles pendant les périodes de 5 ans précédant et suivant la légalisation, comparativement aux États témoins. Une analyse distincte qui a comparé les taux avant et après l'ouverture des points de vente de cannabis (étant donné que la légalisation et la mise en vente ont commencé à des moments différents) a constaté une augmentation significative des accidents de la route mortels après l'ouverture des points de vente $(+1,8$ collision mortelle/milliard de milles parcourus, IC à $95 \%$ 0,4-3,7). Une étude de 2020 utilisant des données similaires a observé une augmentation des accidents de la route mortels au Colorado ( $+1,5$ décès par milliard de milles parcourus; $p=0,047)$, mais pas dans l'État de Washington (+0,08 décès/milliard de milles parcourus; $p=0,67$ ) après la légalisation des points de vente de cannabis à des fins récréatives ${ }^{13}$. Les auteurs ont formulé l'hypothèse que des différences entre les États (p. ex., hausse de la densité des points de vente, de l'utilisation du cannabis et du tourisme lié au cannabis) pourraient avoir contribué à l'augmentation observée des accidents de la route mortels au Colorado seulement. Une autre étude réalisée en 2020 a observé une augmentation des accidents de la route mortels (+2,1 décès/milliard de milles parcourus; IC à $95 \%$ 1,3-3,0) dans 4 États (Colorado, Washington, Oregon et Alaska) après la légalisation de la vente au détail de cannabis à des fins récréatives, comparativement à 20 États témoins où l'utilisation du cannabis à des fins récréatives ${ }^{14}$ ou médicales n'avait pas été légalisée ${ }^{14}$

Dans l'ensemble, les données disponibles tirées d'études écologiques suggèrent que la légalisation du cannabis à des fins récréatives serait associée à une augmentation relative légère, mais non négligeable du nombre d'accidents de la route mortels aux États-Unis. Cette augmentation pourrait être temporaire ou permanente. Il faudra réaliser d'autres analyses rigoureuses à mesure que plus de données seront disponibles.

\section{Les mesures visant à détecter et à prévenir la conduite avec facultés affaiblies par le cannabis au Canada seront-elles efficaces?}

Au Canada, l'adoption de mesures législatives et de santé publique prudentes pourrait éviter une hausse de la conduite avec facultés affaiblies par le cannabis et de ses conséquences après la légalisation du cannabis à usage récréatif. Cela dit, une réponse multidisciplinaire s'impose pour une prévention et une dissuasion optimales ${ }^{15}$. Il existe une incertitude substantielle quant à la façon optimale d'utiliser les mesures et les outils disponibles pour détecter la conduite avec facultés affaiblies par le cannabis².

Le Canada utilise actuellement des évaluations psychomotrices pour la détection des facultés affaiblies par des drogues lors de 
contrôles routiers ${ }^{3}$. Elles incluent des tests de sobriété normalisés : se tenir debout sur un pied, le test du nystagmus du regard horizontal et marcher et se retourner ${ }^{16}$. Ces tests ont été mis au point pour évaluer les facultés affaiblies par l'alcool, et leur validité pour la détection des facultés affaiblies par le cannabis n'est pas démontrée ${ }^{3}$. Après l'échec au test de sobriété normalisé, un officier dûment formé, par exemple un expert en reconnaissance des drogues, procédera à des tests neurologiques et ophtalmologiques plus complexes pour évaluer de potentielles facultés affaiblies. Toutefois, ces experts sont peu nombreux au Canada, car la formation est coûteuse et demande du temps, et le territoire à couvrir est trop vaste pour l'effectif ${ }^{2,17}$. De plus, les experts en reconnaissance des drogues n'ont pas de formation médicale, et la précision avec laquelle ils peuvent détecter les facultés affaiblies et les attribuer à une classe de drogues en particulier varie ${ }^{18}$. La validité du test de sobriété normalisé et du programme d'expertise en reconnaissance de drogues pour ce qui est des facultés affaiblies par le cannabis demeure incertaine et d'autres méthodes méritent d'être explorées pour compléter ou remplacer ces approches.

Le dosage biochimique du cannabis dans le sang, l'urine ou la salive pourrait être utilisé en plus des évaluations psychomotrices pour mesurer à quel point les facultés sont affaiblies. Le projet de loi C-46 (2018) permet à la police d'effectuer un dépistage du THC dans la salive lors d'un contrôle routier avec plusieurs dispositifs actuellement approuvés et d'autres en cours d'évaluation ${ }^{19}$. Ce projet de loi précise également les seuils de THC en lien avec la conduite $(2-5 \mathrm{ng} / \mathrm{mL}$ et $\geq 5 \mathrm{ng} / \mathrm{mL})$ avec des amendes minimales pour conduite avec facultés affaiblies et des amendes plus sévères en présence de seuils de THC inférieurs si l'alcool est en cause $(\geq 2,5 \mathrm{ng} / \mathrm{mL}$ de THC avec concentration d'alcool dans le sang $\geq 0,5 \mathrm{mg} / \mathrm{mL})^{19}$. Les provinces et les territoires peuvent appliquer des politiques plus strictes (le Québec et la Saskatchewan ont des politiques de tolérance zéro et prévoient des amendes pour toute trace détectable de THC) ${ }^{20,21}$. L'applicabilité de ces limites et leur capacité à résister aux contestations judiciaires restent à vérifier, puisqu'elles ne se fondent pas sur une corrélation démontrée entre les taux de THC et la conduite avec facultés affaiblies ${ }^{17,22}$.

Par ailleurs, le tétrahydrocannabinol est liposoluble, ce qui signifie qu'il se retrouve rapidement dans le cerveau et d'autres organes, où ses métabolites peuvent persister pendant des périodes prolongées, ce qui rend difficile une mesure biochimique précise des facultés affaiblies ${ }^{3}$. En outre, le degré relatif d'affaiblissement des facultés associé à une concentration de THC donnée pourrait différer d'un individu à l'autre en raison de différences individuelles de tolérance ${ }^{23}$, et les utilisateurs quotidiens de cannabis de longue date pourraient continuer de présenter des taux détectables de THC dans la salive ou l'urine après des semaines, voire des mois d'abstinence ${ }^{24,25}$. Les diverses formes de consommation du cannabis (p. ex., inhalation, vapotage, produits comestibles) rendent également les mesures plus difficiles, étant donné que chacune a une courbe de concentration de THC différente dans les liquides organiques ${ }^{2}$. Une amélioration substantielle de la précision des mesures biochimiques actuelles de détection des facultés affaiblies par le cannabis sera nécessaire pour assurer la sécurité du public et protéger les droits des consommateurs légaux de cannabis.
Bien qu'une incertitude demeure quant à la précision des tests, les messages rappelant au public que la conduite avec facultés affaiblies est détectable et passible d'amendes, eux, seront probablement efficaces p our prévenir la conduite a vec facultés affaiblies par le cannabis. Les campagnes de sensibilisation entourant les lois relatives à l'alcoolémie au volant entrées en vigueur entre 1982 et 2000 dans des États américains aurait réduit de $14 \%$ à $15 \%$ le nombre de collisions liées à l'alcool ${ }^{26,27}$. L'établissement de limites fédérales pour la conduite après consommation de THC pourrait donc s'avérer utile et être plus bénéfique pour les Canadiens que les stratégies dissuasives adoptées aux États-Unis. Parmi les 11 États américains qui avaient des lois en vigueur sur l'utilisation du cannabis à des fins récréatives en 2020 , seulement 4 préconisaient des limites visant expressément la conduite sous l'influence du cannabis ${ }^{8}$.

D'autres stratégies de prévention incluent l'application de politiques de tolérance zéro pour les jeunes conducteurs, de contrôles routiers ponctuels et de sanctions administratives (p. ex., contraventions, amendes, saisie de véhicules, suspension du permis de conduire) dès le verdict de culpabilité de conduite avec facultés affaiblies ${ }^{2}$. Une récente revue a conclu que lorsque des techniques policières proactives sont fortement publicisées et visibles, elles peuvent avoir un effet immédiat et considérable sur la réduction de la conduite avec facultés affaiblies ${ }^{28}$.

\section{Les professionnels de la santé peuvent-ils contribuer à prévenir la conduite avec facultés affaiblies?}

En contexte de soins primaires, de brèves interventions se sont révélées efficaces pour réduire la consommation d'alcool problématique et d'autres comportements à risque ${ }^{29}$, et elles pourraient être adaptées pour la conduite sous l'influence du cannabis. Nous n'avons pas trouvé de données probantes indiquant que les risques pour la conduite sont différents chez les personnes qui utilisent du cannabis à des fins thérapeutiques plutôt que récréatives. Par conséquent, les mêmes renseignements devront être transmis aux 2 groupes.

Les Recommandations canadiennes pour l'usage du cannabis à moindre risque recommandent plusieurs mesures fondées sur des données probantes que les patients devraient appliquer pour réduire le risque de préjudices graves (annexe 1, accessible en anglais au www.cmaj.ca/lookup/doi/10.1503/cmaj.191032/tab -related-content) ${ }^{30}$. Ces étapes incluent : choisir des produits moins puissants, se limiter à un usage occasionnel, éviter de fumer le cannabis et utiliser plutôt les vaporisateurs ou les produits comestibles (notons toutefois que selon les recommandations, les produits comestibles sont plus sécuritaires pour la santé respiratoire, mais que comme l'effet psychoactif est retardé, il est plus facile d'en consommer de fortes doses, qui auraient alors un effet plus marqué sur les facultés ${ }^{31}$. Les recommandations mettent le lecteur en garde contre la conduite après la consommation de cannabis (surtout combiné à l'alcool), et suggèrent d'attendre au moins 6 heures avant de prendre le volant ${ }^{30}$.

L'encadré 1 présente une liste d'énoncés fondés sur des données probantes qui pourraient être utiles aux professionnels de la 
Encadré 1 : Conduite avec facultés affaiblies par le cannabis au Canada

- L'utilisation du cannabis altère l'aptitude à conduire et la capacité d'effectuer les tâches requises, comme ne pas dévier de sa trajectoire, maintenir une vitesse constante, prendre de bonnes décisions sur la route et éviter les dangers ${ }^{1,2}$.

- La consommation concomitante d'alcool et de cannabis altère davantage l'aptitude à conduire que l'utilisation de l'un ou l'autre seul'5.

- La consommation dans un véhicule (même à l'arrêt) est interdite. Dans un véhicule, le cannabis doit être placé dans un endroit clos hors de la portée du conducteur et des passagers (p. ex., dans le coffre arrière) ${ }^{3}$.

- La conduite après la consommation de drogues est illégale. La police dispose de nombreux outils pour détecter la conduite avec facultés affaiblies, y compris des tests de sobriété et des tests de salive lors de contrôles routiers, des analyses sanguines et l'évaluation par un expert en reconnaissance des drogues. Les amendes pour conduite avec facultés affaiblies par la drogue incluent une amende maximale de $1000 \$$ pour un taux de THC $>2 \mathrm{ng} / \mathrm{mL}$, mais $<5 \mathrm{ng} / \mathrm{mL}$ ou, si les taux de THC sont plus élevés $(\geq 5 \mathrm{ng} / \mathrm{mL})^{4}$ :

- Première offense : amende obligatoire minimum de 1000 ; et possibilité de 10 ans d'incarcération;

- Deuxième offense : incarcération minimum obligatoire de 30 jours (maximum 10 ans);

- Troisième offense : incarcération minimum obligatoire de 120 jours (maximum 10 ans).

- Le cannabis affecte chaque personne différemment, et ses effets dépendent du produit utilisé, du mode d'utilisation et de la quantité consommée. Avec les produits comestibles en particulier, les effets subjectifs peuvent prendre plus de temps à se manifester, ce qui pourrait entraîner une consommation involontairement supérieure aux intentions. Lorsqu'on consomme du cannabis, ses effets entraînent une inaptitude à juger de son aptitude ${ }^{2}$.

- Ne prenez pas de risques. Si vous avez l'intention de consommer, préparez-vous en optant pour un mode de transport différent ou en consommant bien avant de prendre le volant (au moins 6 heures) $)^{2}$.

santé qui souhaitent mettre leurs patients en garde contre l'utilisation du cannabis et la conduite "sous influence ». Les patients pourraient ignorer que la consommation dans un véhicule, même à l'arrêt, est interdite, et que tout produit de cannabis présent dans un véhicule doit être hors de la portée du conducteur (p. ex., dans le coffre arrière) ${ }^{32}$. Les lignes directrices nationales et les lois provinciales enjoignent les médecins d'informer leurs patients que s'ils veulent prendre le volant après avoir consommé du cannabis, sous quelque forme que ce soit (y compris les cannabinoïdes synthétiques), ils pourraient encore présenter des taux excédant les limites de THC autorisées 6 heures après la consommation (particulièrement au Québec et en Saskatchewan, qui ont des politiques de tolérance zéro à l'égard du THC) ${ }^{17,33,34}$ et de leur rappeler les amendes prévues pour tout dépassement des limites fixées (indépendamment du degré d'affaiblissement des facultés ${ }^{35}$. Bien que le pic plasmatique de concentration de THC apparaisse en général dans les 5-30 minutes suivant la consommation et décline graduellement après 2-4 heures, les facultés peuvent demeurer affaiblies 3-6 heures après la consommation, selon la voie d'administration et les particularités individuelles. Les professionnels de la santé devraient aussi connaître les dispositions concernant les exigences de déclaration discrétionnaires ou obligatoires dans leur province ou leur territoire pour ce qui est de la conduite après avoir consommé des substances ${ }^{36}$, même si l'état des connaissances en ce qui concerne l'aptitude à conduire, particulièrement chez les utilisateurs réguliers et de longue date de cannabis, n'apporte pas de certitude absolue.

\section{Conclusion}

Selon des données américaines, il existe un risque léger, mais non négligeable d'augmentation du nombre d'accidents de la route fatals après la légalisation du cannabis à des fins récréatives au Canada, qui pourrait entraîner environ 308 décès additionnels annuellement ${ }^{8}$, et des milliers d'autres blessures non mortelles. Toutefois, l'appalication de mesures de prévention de la conduite avec facultés affaiblies et de sensibilisation, y compris sur les limites fixées par le gouvernement fédéral pour la conduite sous l'effet du THC, et les campagnes de publicité au sujet de ces limites, pourrait contribuer à prévenir une augmentation potentielle de la conduite avec facultés affaiblies par le cannabis au Canada. Les professionnels de la santé devraient donner à leurs patients qui utilisent du cannabis des conseils clairs et fondés sur des données probantes pour une utilisation plus sécuritaire.

\section{Références}

1. Busardo FP, Pellegrini M, Klein J, et al. Neurocognitive correlates in driving under the influence of cannabis. CNS Neurol Disord Drug Targets 2017;16: 534-40.

2. A framework for the legalization and regulation of cannabis in Canada: The final report of the Task Force on Cannabis Legalization and Regulation. Ottawa: Health Canada; 2016. Accessible ici : http://healthycanadians.gc.ca/task-force -marijuana-groupe-etude/framework-cadre/alt/framework-cadre-eng.pdf (consulté le 5 juill. 2019).

3. Capler R, Bilsker D, Van Pelt K, et al. Cannabis use and driving: evidence review. Burnaby (BC): Canadian Drug Policy Coalition; 2017. Accessible ici : http://drugpolicy.ca/wp-content/uploads/2016/11/CDPC_Cannabis-and-Driving _Evidence-Review-Full_Jan31-2017_FINAL.pdf (consulté le 20 juin 2019).

4. Elvik R. Risk of road accident associated with the use of drugs: a systematic review and meta-analysis of evidence from epidemiological studies. Accid Anal Prev 2013;60:254-67.

5. National Cannabis Survey, first quarter 2019. Ottawa: Statistics Canada; 2019 Accessible ici : https://www150.statcan.gc.ca/n1/daily-quotidien/190502/ dq190502a-eng.htm (consulté le 31 mai 2019)

6. Canadian Cannabis Survey 2018 Summary. Ottawa: Government of Canada; 2018. Accessible ici : www.canada.ca/en/services/health/publications/drugs -health-products/canadian-cannabis-survey-2018-summary.html (consulté le 4 juill. 2019).

7. Wettlaufer A, Florica RO, Asbridge M, et al. Estimating the harms and costs of cannabis-attributable collisions in the Canadian provinces. Drug Alcohol Depend 2017;173:185-90.

8. Windle SB, Eisenberg MJ, Reynier P, et al. Association between legalization of recreational cannabis and fatal motor vehicle collisions in the United States: an ecological study. CMAJ Open 2021;9:E233-41.

9. Canadian motor vehicle traffic collision statistics: 2018. Ottawa: Government of Canada [Transport Canada]; 2019. Accessible ici : https://tc.canada.ca/en/canadian -motor-vehicle-traffic-collision-statistics-2018 (consulté le 5 juill. 2019). 
10. Tefft BC, Arnold LS, Grabowski JG. Prevalence of marijuana involvement in fatal crashes: Washington, 2010-2014. Washington (D.C): AAA Foundation for Traffic Safety; 2016. Accessible ici : https://aaafoundation.org/wp-content/ uploads/2017/12/PrevalenceOfMarijuanalnvolvement.pdf (consulté le 5 juill. 2019).

11. Lane TJ, Hall W. Traffic fatalities within US states that have legalized recreational cannabis sales and their neighbours. Addiction 2019;114:847-56.

12. Aydelotte JD, Mardock AL, Mancheski CA, et al. Fatal crashes in the 5 years after recreational marijuana legalization in Colorado and Washington. Accid Anal Prev 2019;132:105284.

13. Santaella-Tenorio J, Wheeler-Martin K, DiMaggio CJ, et al. Association of recreational cannabis laws in Colorado and Washington State with changes in traffic fatalities, 2005-2017. JAMA Intern Med 2020;180:1061-8.

14. Kamer RS, Warshafsky S, Kamer GC. Change in traffic fatality rates in the first 4 states to legalize recreational marijuana. JAMA Intern Med 2020;180:1119-20.

15. Marillier M, Verstraete AG. Driving under the influence of drugs. WIREs Forensic Sci 2019;1:e1326.

16. Bosker WM, Theunissen EL, Conen S, et al. A placebo-controlled study to assess Standardized Field Sobriety Tests performance during alcohol and cannabis intoxication in heavy cannabis users and accuracy of point of collection testing devices for detecting THC in oral fluid. Psychopharmacology (Berl) 2012;223:439-46.

17. Peaire A, Filbert A, Smith D, et al. Report on drug per se limits. Ottawa: Canadian Society of Forensic Sciences; 2017. Accessible ici : www.csfs.ca/wp-content/ uploads/2017/09/Report-on-Drug-Per-Se-Limit.pdf (consulté le 20 juin 2019).

18. Beirness DJ, LeCavalier J, Singhal D. Evaluation of the Drug Evaluation and Classification program: a critical review of the evidence. Traffic Inj Prev 2007;8:368-76.

19. Bill C-46. Ottawa: Parliament of Canada; 2018. Accessible ici : www.parl.ca/ DocumentViewer/en/42-1/bill/C-46/royal-assent (consulté le 26 févr. 2019).

20. Regulation of cannabis in Québec. Québec City: Government of Quebec; 2019. Accessible ici : https://encadrementcannabis.gouv.qc.ca/en/ (consulté le 3 juin 2019).

21. Cannabis in Saskatchewan. Regina: Government of Saskatchewan; 2019. Accessible ici : www.saskatchewan.ca/government/cannabis-in-saskatchewan (consulté le 3 juin 2019).

22. Roth A. The uneasy case for marijuana as chemical impairment under a sciencebased jurisprudence of dangerousness. California Law Review 2015;103: 841-918.

23. Desrosiers NA, Ramaekers JG, Chauchard E, et al. Smoked cannabis' psychomotor and neurocognitive effects in occasional and frequent smokers. J Anal Toxicol 2015;39:251-61.
24. Bosker WM, Karschner EL, Lee D, et al. Psychomotor function in chronic daily cannabis smokers during sustained abstinence. PLoS One 2013;8:e53127.

25. Ellis GM Jr, Mann MA, Judson BA, et al. Excretion patterns of cannabinoid metabolites after last use in a group of chronic users. Clin Pharmacol Ther 1985; 38:572-8.

26. Tippetts AS, Voas RB, Fell JC, et al. A meta-analysis of. 08 BAC laws in 19 jurisdictions in the United States. Accid Anal Prev 2005;37:149-61.

27. Villaveces A, Cummings P, Koepsell TD, et al. Association of alcohol-related laws with deaths due to motor vehicle and motorcycle crashes in the United States, 1980-1997. Am J Epidemiol 2003;157:131-40.

28. Fell JC. Approaches for reducing alcohol-impaired driving: evidence-based legislation, law enforcement strategies, sanctions, and alcohol-control policies. Forensic Sci Rev 2019;31:161-84.

29. Final recommendation statement: unhealthy alcohol use in adolescents and adults: screening and behavioral counseling interventions. Rockville (MD): U.S. Preventive Services Task Force; 2018. Accessible ici : www.uspreventiveservicestaskforce. org/Page/Document/RecommendationStatementFinal/unhealthy-alcohol-use -in-adolescents-and-adults-screening-and-behavioral-counseling-interventions (consulté le 23 oct. 2019).

30. Fischer B, Russell C, Sabioni P, et al. Lower-risk cannabis use guidelines: a comprehensive update of evidence and recommendations. Am J Public Health 2017;107:e1-12.

31. Grewal JK, Loh LC. Health considerations of the legalization of cannabis edibles. CMAJ 2020;192:E1-2.

32. Lancione S, Wade K, Windle SB, et al. Non-medical cannabis in North America: an overview of regulatory approaches. Public Health 2020;178:7-14.

33. Huestis MA, Milman G, Mendu DR, et al. Evaluation of the on-site Draeger DrugTest 5000 in occasional and chronic frequent smokers following controlled cannabis smoking. Semantic Scholar; 2013. Accessible ici : www.semanticscholar. org/paper/Evaluation-of-the-on-site-Draeger-DrugTest-5000-in-Huestis-Milman /8991d00dc75bb423f92bbfe69d3bb64ef31fb4ec\#citing-papers (consulté le 3 oct. 2019).

34. Tuv SS, Krabseth $\mathrm{H}$, Karinen $\mathrm{R}$, et al. Prevalence of synthetic cannabinoids in blood samples from Norwegian drivers suspected of impaired driving during a seven weeks period. Accid Anal Prev 2014;62:26-31.

35. Impaired driving laws. Ottawa: Department of Justice; 2019. Accessible ici : www.justice.gc.ca/eng/cj-jp/sidl-rlcfa/ (consulté le 4 juill. 2019).

36. Hit the brakes: Do you need to report your patient's fitness to drive? Ottawa: Canadian Medical Protective Association; 2019. Accessible ici : www.cmpa-acpm. ca/en/advice-publications/browse-articles/2019/hit-the-brakes-do-you-need-to -report-your-patients-fitness-to-drive (consulté le 20 nov. 2019).

\section{Intérêts concurrents : Aucun déclaré.}

Cet article a été révisé par des pairs.

Affiliations : Institut Lady Davis (Windle, Sequeira, Filion, Thombs, Reynier, Grad, Ells, Eisenberg), Hôpital général juif; Département d'épidémiologie, de biostatistique et de santé du travail (Windle, Filion, Thombs, Eisenberg), Université McGill; Faculté de médecine (Filion, Thombs, Eisenberg), Université McGill; Départements de psychiatrie, de psychologie et de psychologie de l'enseignement et du counseling (Thombs), Université McGill; Département de médecine familiale (Grad, Ells), Université McGill; Unité d'éthique biomédicale, Division de médecine expérimentale et Département des sciences sociales en médecine (Ells), Université McGill; Département de cardiologie (Eisenberg), Hôpital général juif, Montréal, Qc.
Collaborateurs : Sarah Windle et Crystal Sequeira ont collaboré à parts égales en tant que premières auteures. Sarah Windle, Mark Eisenberg et Kristian Filion ont contribué à la conception et à la modélisation des travaux. Sarah Windle et Crystal Sequeira ont rédigé ensemble l'ébauche du manuscrit. Tous les auteurs ont révisé de façon critique le contenu intellectuel important du manuscrit; ils ont donné leur approbation finale pour la version destinée à être publiée et assument l'entière responsabilité de tous les aspects du travail.

Financement : Cette étude a été financée par les Instituts de recherche en santé du Canada (SHI-155407). Sarah Windle est titulaire d'une bourse d'études supérieures MaysieMacSporran, ainsi que d'une bourse Alma Mater Fellowship et d'un prix d'excellence des cycles supérieurs de l'Université McGill.
Kristian Filion est chercheur-boursier Senior du Fonds de recherche du Québec - Santé (FRQS) et titulaire de la bourse William Dawson de l'Université McGill. Brett Thombs est chercheur-boursier du FRQS.

Propriété intellectuelle du contenu : Il s'agit d'un article en libre accès distribué conformément aux modalités de la licence Creative Commons Attributions (CC BY-NC-ND 4.0), qui permet l'utilisation, la diffusion et la reproduction dans tout médium à la condition que la publication originale soit adéquatement citée, que l'utilisation se fasse à des fins non commerciales (c.-à-d., recherche ou éducation) et qu'aucune modification ni adaptation n'y soit apportée. Voir : https://creativecommons.org/ licenses/by-nc-nd/4.0/deed.fr.

Correspondance : Mark Eisenberg, mark.eisenberg@mcgill.ca 